\title{
ANALYTICAL INVESTIGATION ON THE DYNAMIC APERTURES OF CIRCULAR ACCELERATORS
}

\author{
J. Gao, LAL, B.P. 34, F-91898 Orsay cedex, France
}

\section{Abstract}

In this paper by considering delta function sextupole and octupole perturbations and using difference action-angle variable equations, analytical formulae for the dynamic aperture of circular accelerators are derived based on the Chirikov criterion of the onset of stochastic motions.

\section{INTRODUCTION}

One of the preoccupations of the circular accelerator designers is to estimate the influence of nonlinear forces on the single particle's motion. Even though the nonlinear forces compared with the linear ones are usually very small, what is observed in reality, however, is that when the amplitudes of the transverse oscillation of a particle are large enough, the transverse motions might become unstable and the particle itself will finally be lost on the vacuum chamber. In the following sections we will show how the nonlinear forces limit the dynamic apertures and what is the relation between them.

\section{DYNAMIC APERTURES DUE TO NONLINEAR RESONANCES AND STOCHASTIC MOTIONS}

In this section a necessary distinction will be made between two essentially different cases: a proton machine and an electron one. The reason is simple. In the first case there is no dissipative forces (which is generally true) and the particle's motion can be described in the frame of Hamiltonian system. In the second case, however, one has to take into account of the synchrotron radiation damping effect.

\subsection{Proton storage ring}

To start with we consider the linear horizontal motion of a particle assuming that the magnetic field is only transverse and there are no screw fields. The Hamiltonian can be expressed as

$$
H=\frac{p^{2}}{2}+\frac{K(s)}{2} x^{2}
$$

where $x$ denotes normal plane coordinate, $p=d x / d s$, and $K(s)$ is a periodic function satisfying the relation

$$
K(s)=K(s+L)
$$

where $L$ is the circumference of the ring. The solution of the deviation, $x$, is found to be

$$
x=\sqrt{\epsilon_{x} \beta_{x}(s)} \cos \left(\phi(s)+\phi_{0}\right)
$$

where

$$
\phi(s)=\int_{0}^{s} \frac{d s}{\beta_{x}(s)}
$$

As an essential step towards further discussion on the motions under nonlinear perturbation forces, we introduce action-angle variables and the Hamiltonian expressed in these new variables [1]:

$$
\begin{gathered}
\Psi=\int_{0}^{s} \frac{d s^{\prime}}{\beta_{x}\left(s^{\prime}\right)}+\phi_{0} \\
J=\frac{\epsilon_{x}}{2}=\frac{1}{2 \beta_{x}(s)}\left(x^{2}+\left(\beta_{x}(s) x^{\prime}-\frac{\beta_{x}^{\prime} x}{2}\right)^{2}\right) \\
H(J, \Psi)=\frac{J}{\beta_{x}(s)}
\end{gathered}
$$

Since the $H(J, \Psi)=J / \beta_{x}(s)$ is still a function of the independent variable, $s$, we will make another canonical transformation to freeze the new Hamiltonian:

$$
\begin{gathered}
\Psi_{1}=\Psi+\frac{2 \pi \nu}{L}-\int_{0}^{s} \frac{d s^{\prime}}{\beta_{x}\left(s^{\prime}\right)} \\
J_{1}=J \\
H_{1}=\frac{2 \pi \nu}{L} J_{1}
\end{gathered}
$$

Before going on further, let's remember the relation between the last action-angle variables and the particle deviation $x$ :

$$
x=\sqrt{2 J_{1} \beta_{x}(s)} \cos \left(\Psi_{1}-\frac{2 \pi \nu}{L} s+\int_{0}^{s} \frac{d s^{\prime}}{\beta_{x}\left(s^{\prime}\right)}\right)
$$

Having well prepared we start our journey to find out the limitations of the nonlinear forces on the stability of the particle's motion. To facilitate the analytical treatment of this complicated problem we consider at this stage only sextupoles and octupoles (no screw terms) and assume that the contributions from the sextupoles and octupoles in a ring can be made equivalent to a point sextupole and a point octupole. The perturbed Hamiltonian can be thus expressed:

$$
\begin{aligned}
\mathcal{H}=\frac{p^{2}}{2}+ & \frac{K(s)}{2} x^{2}+\frac{1}{3 ! B \rho} \frac{\partial^{2} B_{z}}{\partial x^{2}} x^{3} L \sum_{k=-\infty}^{\infty} \delta(s-k L) \\
& +\frac{1}{4 ! B \rho} \frac{\partial^{3} B_{z}}{\partial x^{3}} x^{4} L \sum_{k=-\infty}^{\infty} \delta(s-k L)
\end{aligned}
$$

where $\rho$ is the radius of curvature. Representing eq. 12 by action-angle variables ( $J_{1}$ and $\left.\Psi_{1}\right)$, and using

$$
B_{z}=B_{0}\left(1+x b_{1}+x^{2} b_{2}+x^{3} b_{3}\right)
$$


one has

$$
\begin{aligned}
H= & \frac{2 \pi \nu}{L} J_{1}+\frac{\left(2 J_{1} \beta_{x}\left(s_{1}\right)\right)^{3 / 2}}{3 \rho} b_{2} L \cos ^{3} \Psi_{1} \sum_{k=-\infty}^{\infty} \delta(s-k L) \\
& +\frac{\left(J_{1} \beta_{x}\left(s_{2}\right)\right)^{2}}{\rho} b_{3} L \cos ^{4} \Psi_{1} \sum_{k=-\infty}^{\infty} \delta(s-k L)
\end{aligned}
$$

where $s_{1}$ and $s_{2}$ are just used to differentiate the locations of the sextupole and the octupole perturbations. By virtue of Hamiltonian one gets the differential equations for $\Psi_{1}$ and $J_{1}$

$$
\begin{gathered}
\frac{d J_{1}}{d s}=-\frac{\partial \mathcal{H}}{\partial \Psi_{1}} \\
\frac{d \Psi_{1}}{d s}=\frac{\partial \mathcal{H}}{\partial J_{1}} \\
\frac{d J_{1}}{d s}=-\frac{\left(2 J_{1} \beta_{x}\left(s_{1}\right)\right)^{3 / 2}}{3 \rho} b_{2} L \frac{d \cos ^{3} \Psi_{1}}{d \Psi_{1}} \sum_{k=-\infty}^{\infty} \delta(s-k L) \\
-\frac{\left(J_{1} \beta_{x}\left(s_{2}\right)\right)^{2}}{\rho} b_{3} L \frac{d \cos ^{4} \Psi_{1}}{d \Psi_{1}} \sum_{k=-\infty}^{\infty} \delta(s-k L) \\
\frac{d \Psi_{1}}{d s}=\frac{2 \pi \nu}{L}+\frac{2 \beta_{x}^{2}\left(s_{2}\right)}{\rho} J_{1} b_{3} L \cos ^{4} \Psi_{1} \sum_{k=-\infty}^{\infty} \delta(s-k L) \\
+\frac{\sqrt{2} J_{1}^{1 / 2} \beta_{x}\left(s_{1}\right)^{3 / 2}}{\rho} b_{2} L \cos ^{3} \Psi_{1} \sum_{k=-\infty}^{\infty} \delta(s-k L)
\end{gathered}
$$

Now it is the moment to change this differential equations to the difference equations which is suitable to analyse the possibilities of the onset of stochasticity [2][3]. Since the perturbations have a natural periodicity of $L$ we will sample the dynamic quantities at a sequence of $s_{i}$ with constant interval $L$ assuming that the characteristic time between two consecutive adiabatic invariance breakdown intervals is shorter than $L / c$. The differential equations in eqs. 17 and 18 are reduced to

$$
\begin{aligned}
& \overline{J_{1}}=\overline{J_{1}}\left(\Psi_{1}, J_{1}\right) \\
& \overline{\Psi_{1}}=\overline{\Psi_{1}}\left(\Psi_{1}, J_{1}\right)
\end{aligned}
$$

where the bar stands for the next sampled value after the corresponding unbared previous value, or explicitly,

$$
\begin{gathered}
\overline{J_{1}}=J_{1}-\frac{\left(2 J_{1} \beta_{x}\left(s_{1}\right)\right)^{3 / 2}}{3 \rho} b_{2} L \frac{d \cos ^{3} \Psi_{1}}{d \Psi_{1}} \\
-\frac{\left(J_{1} \beta_{x}\left(s_{2}\right)\right)^{2}}{\rho} b_{3} L \frac{d \cos ^{4} \Psi_{1}}{d \Psi_{1}} \\
\overline{\Psi_{1}}=\Psi_{1}+2 \pi \nu+\frac{\sqrt{2} \beta_{x}\left(s_{1}\right)^{3 / 2}{\overline{J_{1}}}^{1 / 2}}{\rho} b_{2} L \cos ^{3} \Psi_{1} \\
+\frac{2 \beta_{x}\left(s_{2}\right)^{2}}{\rho} \overline{J_{1}} b_{3} L \cos ^{4} \Psi_{1}
\end{gathered}
$$

Eqs. 21 and 22 are the basic difference equations to study the nonlinear resonance and the onset of stochasticities considering sextupole and octupole perturbations. By using trigonometric relation

$$
\cos ^{m} \theta \cos n \theta=2^{-m} \sum_{r=0}^{m} \frac{m !}{(m-r) ! r !} \cos (n-m+2 r) \theta
$$

one has

$$
\begin{gathered}
\cos ^{3} \theta=\frac{2}{2^{3}}(\cos 3 \theta+3 \cos \theta) \\
\cos ^{4} \theta=\frac{1}{2^{4}}\left(\cos 4 \theta+4 \cos 2 \theta+\frac{4 !}{((4 / 2) !)^{2}}\right)
\end{gathered}
$$

Apparently, the right hand sides of eqs. 21 and 22 contain sinusoidal functions of phases, $\Psi_{1}, 2 \Psi_{1}, 3 \Psi_{1}$, and $4 \Psi_{1}$. If the tune $\nu$ is far from the resonance lines $\nu=m / n$, where $m$ and $n$ are integers $(n=1,2,3$, and 4 for this specific problem), the invariant tori of the unperturbed motion are preserved under the presence of the small perturbations by virtue of the Kolmogorov-Arnold-Moser (KAM) theorem. If, however, $\nu$ is close to the above mentioned resonance line, the situation is getting complicated and under some conditions the KAM invariant tori can be broken. Taking the third order resonance, $m / 3$, for example, we keep only the sinusoidal function with phase $3 \Psi_{1}$ in eq. 21 and the dominant phase independent nonlinear term in eq. 22 , and as the result, we have eqs. 21 and 22 reduced to

$$
\begin{gathered}
\overline{J_{1}}=J_{1}+A \sin 3 \Psi_{1} \\
\overline{\Psi_{1}}=\Psi_{1}+B \overline{J_{1}}
\end{gathered}
$$

with

$$
\begin{gathered}
A=\frac{\left(J_{1} \beta_{x}\left(s_{1}\right)\right)^{3 / 2}}{\sqrt{2} \rho} b_{2} L \\
B=\frac{3 \beta_{x}\left(s_{2}\right)^{2}}{4 \rho} b_{3} L
\end{gathered}
$$

where we have dropped the constant phase in eq. 22 . It is helpful to transform eqs. 28 and 29 into the form so-called standard mapping [3] expressed as

$$
\begin{gathered}
\bar{I}=I+K_{0} \sin \theta \\
\bar{\theta}=\theta+\bar{I}
\end{gathered}
$$

with $\theta=3 \Psi, I=3 B J_{1}$ and $K_{0}=3 A B$. By virtue of the Chirikov criterion [3] it is known that when $\left|K_{0}\right| \geq 0.97164$ [4] stochastic motions will appear and the diffusion will occur. Therefore,

$$
\left|K_{0}\right| \leq 1
$$

can be taken as a natural criterion for the determination of the dynamic aperture of the machine. Putting eqs. 28 and 29 into eq. 32, one gets

$$
\left|K_{0}\right|=\frac{9}{4 \sqrt{2}}\left|b_{2} b_{3}\right| \beta_{x}^{2}\left(s_{2}\right) \beta_{x}^{3 / 2}\left(s_{1}\right) J_{1}^{3 / 2} \frac{L^{2}}{\rho^{2}} \leq 1
$$


and consequently, one finds maximum $J_{1}$ corresponding to $m / 3$ resonance

$$
J_{1} \leq J_{\text {max }, m / 3}=\left(\frac{4 \sqrt{2} \rho^{2}}{9\left|b_{2} b_{3}\right| \beta_{x}^{2}\left(s_{2}\right) \beta_{x}\left(s_{1}\right)^{3 / 2} L^{2}}\right)^{2 / 3}
$$

The dynamic aperture of the machine is therefore

$$
\begin{aligned}
& A_{d y n, m / 3}=\sqrt{2 J_{\text {max }, m / 3} \beta_{x}(s)} \\
= & \left(\frac{16 \rho^{2} \beta_{x}(s)^{3 / 2}}{9\left|b_{2} b_{3}\right| \beta_{x}^{2}\left(s_{2}\right) \beta_{x}\left(s_{1}\right)^{3 / 2} L^{2}}\right)^{1 / 3}
\end{aligned}
$$

Eq. 35 gives the dynamic aperture of a sextuple and octupole strength determined case which is believed to be true for the most small emittance electron storage rings. Obviously, the dynamic aperture scales with the one third power of the sextupole and the octupole strength, respectively. If in a storage ring the perturbation from the sextupoles can be omitted, in a similar way one finds the maximum $J_{1}$ corresponding to $m / 4$ resonance mode

$$
J_{1} \leq J_{\text {max }, m / 4}=\frac{2 \rho}{\sqrt{3} \beta_{x}\left(s_{2}\right)^{2} L\left|b_{3}\right|}
$$

and the corresponding dynamic aperture

$$
A_{d y n, m / 4}=\sqrt{2 J_{\max , m / 4} \beta_{x}(s)}=\left(\frac{4 \rho \beta_{x}(s)}{\sqrt{3} \beta_{x}\left(s_{2}\right)^{2} L\left|b_{3}\right|}\right)^{1 / 2}
$$

From eq. 37 one reads that the dynamic aperture, $A_{d y n, m / 4}$, is proportional to the square root of the octupole strength. Usually, one has $A_{d y n, m / 3}<A_{d y n, m / 4}$.

Now I would like to spend some inks on the scenario of those particles whose motions do not satisfy the condition given by eq. 32 . Once a particle begins to execute stochastic motion the phase mixing occurs, and the mapping given by eqs. 30 and 31 can be regarded as a Markov process [5], and in consequence, the possibility distribution function $\mathcal{F}(s, I)$ satisfies the Fokker-Planck equation:

$$
\frac{\partial \mathcal{F}}{\partial s}=-\frac{\partial(\mathcal{A F})}{\partial I}+\frac{1}{2} \frac{\partial^{2}(\mathcal{D F})}{\partial I^{2}}
$$

where $\mathcal{A}=<<\Delta I>>/ L, \mathcal{D}=<<(\Delta I)^{2}>>/ L$, and the notation $\langle<>>$ denotes the average over phase $\theta$. From eq. 30 one knows $\Delta I=K_{0} \sin \theta$, and obviously, one has $\mathcal{A}=0$ and $\mathcal{D}=K_{0}^{2} /(2 L)$. Due to the diffusion, needless to say, the amplitude of the particle's motion is increasing with the distance and finally the particle is lost on the vacuum chamber wall.

\subsection{Electron storage ring}

In an electron storage ring the physical picture is more complicated due to the synchrotron radiation damping. To treat this problem let's resort to the so-called standard dissipative mapping [2] which is different but similar to the standard mapping shown in eqs. 30 and 31 , and expressed as follows:

$$
\begin{gathered}
\bar{I}=\exp (-\Gamma) I+K_{0} \mu \sin \theta \\
\bar{\theta}=\theta+\bar{I}
\end{gathered}
$$

where $\Gamma=\frac{2 L}{\tau_{x} c}, \tau_{x}$ is the damping time of the betatron oscillation in the horizontal direction, and $\mu=(1-$ $\exp (-\Gamma)) / \Gamma$. Apparently, when $\Gamma \rightarrow 0$, eqs. 39 and 40 return to the standard mapping given by eqs. 30 and 31 . The criterion for avoiding the onset of the stochastic motion in the dissipative system is given by

$$
\left|\mu K_{0}\right| \leq 1
$$

The expressions for the dynamic apertures of the electron storage rings corresponding to the two expressions for the proton ones derived in the previous subsection are

$$
\begin{gathered}
A_{d y n, m / 3}=\sqrt{2 J_{\max , m / 3} \beta_{x}(s)} \\
=\left(\frac{16 \rho^{2} \beta_{x}(s)^{3 / 2}}{9 \mu\left|b_{2} b_{3}\right| \beta_{x}^{2}\left(s_{2}\right) \beta_{x}\left(s_{1}\right)^{3 / 2} L^{2}}\right)^{1 / 3}
\end{gathered}
$$

and

$A_{d y n, m / 4}=\sqrt{2 J_{\max , m / 4} \beta_{x}(s)}=\left(\frac{4 \rho \beta_{x}(s)}{\sqrt{3} \beta_{x}\left(s_{2}\right)^{2} L \mu\left|b_{3}\right|}\right)^{1 / 2}$

Eq. 42 has been compared with the numerical dynamic aperture simulation results of ALLADIN [6] and KEK Photon Factory [7], and the satisfactory comparison results are shown in ref. 8.

\section{CONCLUSION}

Considering delta function sextupole and octupole perturbations, analytical expression for the dynamic aperture of a circular machine is obtained by using the Chirikov criterion. It is shown that when the dynamics aperture is sextupole and octupole strength determined which is true for the most cases the dynamic aperture inversely scales with the one third power of the sextupole and octupole strength, respectively. The author thanks J. Le Duff for discussions.

\section{REFERENCES}

[1] R. Ruth, AIP conference proceedings, No. 153, p. 150.

[2] R.Z. Sagdeev, D.A. Usikov, and G.M. Zaslavsky,"Nonlinear Physics, from the pendulum to turbulence and chaos", harwood academic publishers, 1988.

[3] B.V. Chirikov, Physics Reports, Vol. 52, No. 5 (1979), p. 263.

[4] J.M. Greene, J. Math. Phys. 20 (1979), p. 1183.

[5] R.L. Stratonovich, "Topics in the theory of random noise", Vol. 1, Gordon and Breach Science Publishers Inc., 1963.

[6] J. Bridges, et al., Particle Accelerators, Vol. 28 (1990), p. 1.

[7] Y. Kobayash, et al., Proceedings of PAC93 (1993), p. 215, see also: M. Katoh, Particle Accelerators, Vol. 28 (1990), p. 17.

[8] J. Gao, LAL/RT 98-03. 